\title{
Natural frequency of a heavy flexible plate: power law evolution as a function of length
}

\author{
Josué Gilberto Rivas Iñiguez $^{a}$ (D), Maria L. Daza-Torres ${ }^{b}$ id , Abel Palafox González ${ }^{c}$ (D) , Anne Cros $^{a^{*}}$ \\ a Physics Department, Centro Universitario de Ciencias Exactas e Ingenierías, Universidad de Guadalajara, México. \\ E-mail: josue.rivas@alumnos.udg.mx, anne.cros@academicos.udg.mx \\ ${ }^{b}$ Centro de Investigación en Matemáticas, CIMAT A. C., México. E-mail: mdazatorres@cimat.mx \\ c Mathematics Department, Centro Universitario de Ciencias Exactas e Ingenierías, Universidad de Guadalajara, México. \\ E-mail: abel.palafox@academicos.udg.mx \\ * Corresponding author
}

https://doi.org/10.1590/1679-78256479

\begin{abstract}
This theoretical and experimental work deals with the power law evolution followed by the natural frequency $f_{0}$ of a hanging, heavy and flexible plate as a function of its length $L$. When the plate length $L$ is small enough, it behaves as an elastic plate whose weight can be neglected: it is well known that $f_{0}$ evolves as a function of $L^{-2}$. Nevertheless, when the plate length is increased, the mass has to be taken into account, and the previous evolution is not valid anymore. In the case of long elastic plates, $f_{0} \sim L^{-1 / 2}$, just like hanging chains. These two power laws depend on the ratio $L / L_{c}$, where $L_{c}$ is a critical length that writes as a function of the plate mass and the flexural rigidity. After the theory is developed and the plate motion equation is solved using a Galerkin expansion, we find the theoretical evolution of the natural frequencies as a function of length. Experiments were performed with three distinct materials and the natural frequency was systematically measured for a wide length interval. Our data points fit the above-mentioned limit cases and the intermediate case was calculated thanks to our Galerkin expansion.
\end{abstract}

\section{Keywords}

natural frequency, elastic plate, power law

\section{Graphical Abstract}
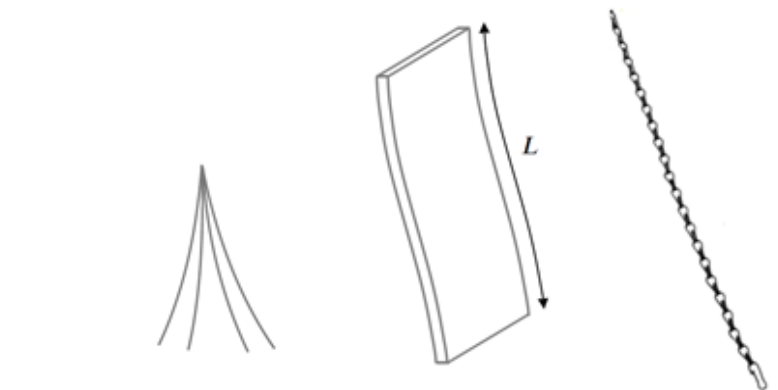

Natural frequency behaves as

Natural frequency behaves as

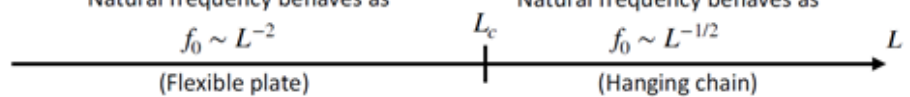

Received March 09, 2021. In revised form April 20, 2021. Accepted April 21, 2021. Available online May 03, 2021. https://doi.org/10.1590/1679-78256479

(c) Latin American Journal of Solids and Structures. ISSN 1679-7825. Copyright (C 2021. This is an Open Access article distributed under the terms of the Creative Commons Attribution License, which permits unrestricted use, distribution, and reproduction in any medium, provided the original work is properly cited. 


\section{INTRODUCTION}

Resonance in structural engineering may have disastrous consequences since it generally leads to high amplitude oscillations that severely damage or destroy the structure. A recent example is the London Millennium Footbridge which had been closed for almost two years because of its lateral oscillations, generated by the footsteps of thousands of pedestrians whose rhythm matched the bridge natural frequency (Newl, 2004). A similar phenomenon may occur in airplanes via the so-called "aeroelastic instability" (Eloy et al., 2007), which originates from a complex interaction between the wing and the airflow (Dungundji et al., 1963). This instability was shown to come from the second natural mode of the structure (Cros and Arellano Castro, 2016).

The natural frequency $f_{0}$ of a flexible plate whose weight is neglected can be easily deduced from the motion equation (Turvey, 1990). In this case, $f_{0}$ depends upon the plate flexural rigidity $E I$, its mass per unit of length and evolves proportionally to $L^{-2}$, where $L$ is the plate length. Now, the case where $E I \rightarrow 0$ corresponds to a hanging chain and it was shown that $f_{0} \sim L^{-1 / 2}$ (Verbin, 2014; Levinson, 1977; Lamb, 1929).

The intermediate case where both gravity and rigidity are taken into account was considered by different authors. In a chronological order, Gregory and Païdoussis (1966) used Rayleigh's method to extract an analytical law for the evolution of $f_{0}$ as a function of L. Païdoussis and Des Trois Maisons (1971) and Schäfer (1985) adopted the Euler beam model in order to estimate how gravity modifies the natural frequency of a flexible beam. Yokoyama (1990) chose to describe the plate thanks to the Timoshenko theory and solved the equation by using the finite element procedure (Xi et al., 2013).

In this study, we first recall the plate motion equation and the nondimensional numbers associated to the problem. The complete system is theoretically solved thanks to a Galerkin expansion and then the experiments are presented. The natural plate frequency is measured for a wide range of sample lengths. The last graphics show how experiments fit the theoretical law and how the nondimensional natural frequency depends only upon a nondimensional length.

\section{THEORY}

The system is shown in Figure 1 . Axis $\mathrm{z}$ is downwards so that the plate is clamped at $z=0$ and free at $z=L$. Lateral oscillations are identified thanks to variable $y$ which depends upon the longitudinal coordinate $z$ and time $t$.
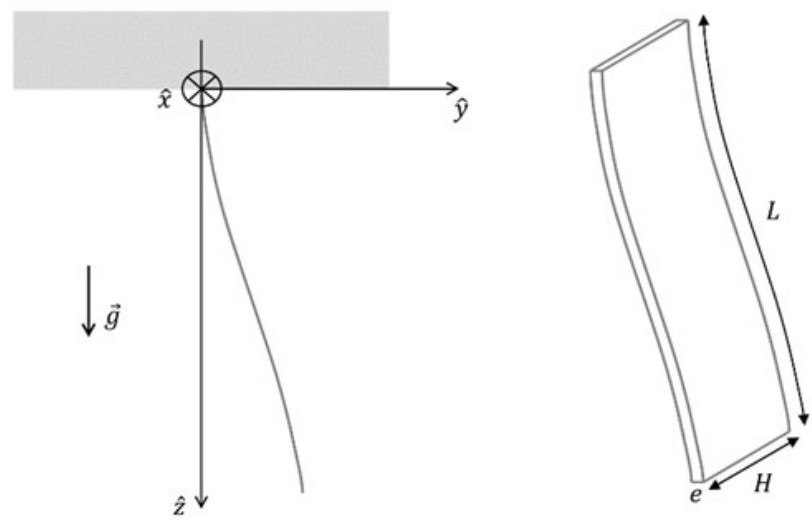

Figure 1: Scheme of our system. The plate is hanging from its top extreme at $z=0$ and $y$ is the transversal deflection. $g$ is gravity. The plate is $L$-long, $H$-wide and $e$-thick.

The complete motion equation is given by the following equation (Païdoussis and Des Trois Maisons, 1971):

$E I \frac{\partial^{4} y}{\partial z^{4}}-\frac{\partial}{\partial z}\left[m_{\ell} g(L-z) \frac{\partial y}{\partial z}\right]+m_{\ell} \frac{\partial^{2} y}{\partial t^{2}}=0$

where $E I$ is the flexural rigidity, $m_{\ell}$ the mass per unit of length and $g$ is gravity. The boundary conditions write as: 


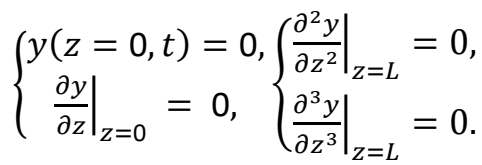

\subsection{Limit case $g=0$}

In the case where the plate's own weight can be neglected, Eq. 1 can be solved by separating variables. The boundary conditions lead to a discrete set of frequency values whose lower frequency is expressed as (Turvey, 1990):

$f_{0}=0.5596 \sqrt{\frac{E I}{m_{\ell}}} \frac{1}{L^{2}}$.

\subsection{Limit case $E I=0$}

If the fourth spatial derivative of Eq. 1 is set to zero, the system corresponds to a hanging chain and the solution is a Bessel function. Once again, the limit conditions at $z=0$, only permit a discrete set of values (Lamb, 1929) in such a way that:

$f_{0}=0.1914 \sqrt{\frac{g}{L}}$.

Let's note that this limit case can be recovered for a flexible plate (for whichEI $\neq 0$ strictly speaking) if its length is high enough. The following section shows indeed that this is possible if $L \gg L_{c}$ where $L_{c}$ is defined by Eq. 6 .

\subsection{Nondimensional equation}

Eq. 1 may be nondimensional by defining the following parameters:

$Y=\frac{y}{L} ; \quad Z=\frac{z}{L} ; \quad T=t \sqrt{\frac{g}{L}}$.

Moreover, a critical length is given by (De Langre et al., 2001):

$L_{c}=\left(\frac{E I}{m_{\ell} g}\right)^{1 / 3}$.

Then Eq. 1 writes as:

$\left(\frac{L_{c}}{L}\right)^{3} \frac{\partial^{4} Y}{\partial Z^{4}}+\frac{\partial Y}{\partial Z}-(1-Z) \frac{\partial^{2} Y}{\partial Z^{2}}+\frac{\partial^{2} Y}{\partial T^{2}}=0$

We see that the plate profile $Y(Z, T)$ only depends upon one parameter which is the nondimensional length $\frac{L}{L_{c}}$. This result is coherent with the Vaschy-Buckingham theorem since there are five independent dimensional parameters: the natural frequency $f_{0}$, the plate length $L$, the flexural rigidity $E I$, the mass per unit of length $m_{\ell}$ and gravity $g$. As a consequence, we can affirm that the nondimensional natural frequency depends only upon a nondimensional length.

\subsection{Intermediate case: our results}

In order to solve the complete equation (Eq. 7) joint with the boundary conditions (Eq. 2), we use the procedure described in Schäfer (1985). This method allows indeed to relate naturally the eigenfrequencies with the plate length without solving the solution $Y(Z, T)$. We assumed a harmonic solution of form:

$Y(Z, T)=\Psi(Z) \sin \left(2 \pi f^{\prime} T\right)$

where $f^{\prime}$ is the natural frequency and $\Psi$ is the corresponding mode shape. Replacing Eq. 8 in Eq. 7 leads to: 
$\Psi^{(4)}(Z)-\alpha\left[(1-Z) \Psi^{\prime}(Z)\right]^{\prime}-\beta \Psi(Z)=0$,

with

$\alpha=\frac{L^{3}}{L_{c}^{3}}$ and $\beta=\left(2 \pi f^{\prime}\right)^{2} \frac{L^{3}}{L_{c}^{3}}$.

Function $\Psi$ is approximated with eigenfunctions $\varphi_{i}$,

$\Psi(Z)=\sum_{i=1}^{n} c_{i} \varphi_{i}(Z)$,

where the $c_{i}$ 's are constant factors to be determined using Galerkin's method. The functions:

$\varphi_{i}(Z):=\cosh \lambda_{i} Z-\cos \lambda_{i} Z+\chi_{i}\left(\sinh \lambda_{i} Z-\sin \lambda_{i} Z\right)$,

with

$\chi_{i}=-\frac{\cosh \lambda_{i}+\cos \lambda_{i}}{\sinh \lambda_{i}+\sin \lambda_{i}}$

are orthogonal and normalized with respect to the inner product $\int_{0}^{1} \varphi_{k} \varphi_{l} d Z=\delta_{k, l}$, where $\delta_{k, l}$ is the Kronecker symbol. These functions satisfy the boundary value problem

$\varphi_{i}^{(4)}(Z)-\lambda_{i}^{4} \varphi_{i}(Z)=0$,

having the same boundary conditions as those presented in Eq. 2 . The eigenvalues $\lambda_{i}$ are solutions of

$\cosh \lambda_{i} \cos \lambda_{i}=-1$,

giving $\lambda_{1}=0.5969, \lambda_{2}=1.4942$, and for the higher values $\lambda_{i} \approx\left(i-\frac{1}{2}\right)$.

The boundary value problem (Eq. 9) together with the homogeneous boundary conditions (Eq. 2) take of the general form:

$M \Psi=\beta \Psi$

where

$M=\frac{d^{4}}{d Z^{4}}-\alpha \frac{d}{d Z}\left((1-Z) \frac{d}{d Z}\right)$.

Inserting Eq. 11 into Eq. 14, we obtain:

$M[\Psi]:=\sum_{i=1}^{n} c_{i}\left(\varphi_{i}^{(4)}(Z)-\alpha\left((1-Z) \varphi_{i}^{\prime}\right)^{\prime}\right)$,

$\beta \Psi:=\beta \sum_{i=1}^{n} c_{i} \varphi(Z)$

Multiplying both sides of the above equation by $\varphi_{k}$

$\sum_{i=1}^{n} c_{i}\left(\varphi_{i}^{(4)}(Z)-\alpha\left((1-Z) \varphi_{i}^{\prime}\right)^{\prime}\right) \varphi_{k}=\beta \sum_{i=1}^{n} c_{i} \varphi_{i}(Z) \varphi_{k}$

for $k=1, \ldots, n$, and applying Galerkin's method leads to the eigenvalue problem: 
$(A-\beta I) C=0$,

where $C=\left(c_{1}, c_{2}, \ldots c_{n}\right)$ is the eigenvector, $I$ is the identity matrix and $\mathrm{A}$ is an $n \times n$ symmetric and positive definite matrix consisting of the entries:

$a_{k l}=\int_{0}^{1} \varphi_{k}\left(\varphi_{l}^{(4)}(Z)-\alpha\left((1-Z) \varphi_{l}^{\prime}\right)^{\prime}\right) d Z$

Upon integration by parts and taking into account both Eq. 13 and the corresponding boundary conditions (Eq. 2):

$a_{k l}=\lambda_{k}^{4} \delta_{k l}+\alpha \int_{0}^{1}(1-Z) \varphi_{k}^{\prime} \varphi_{l}^{\prime} d Z$

Details for solving the above integral can be found in Schäfer (1985).

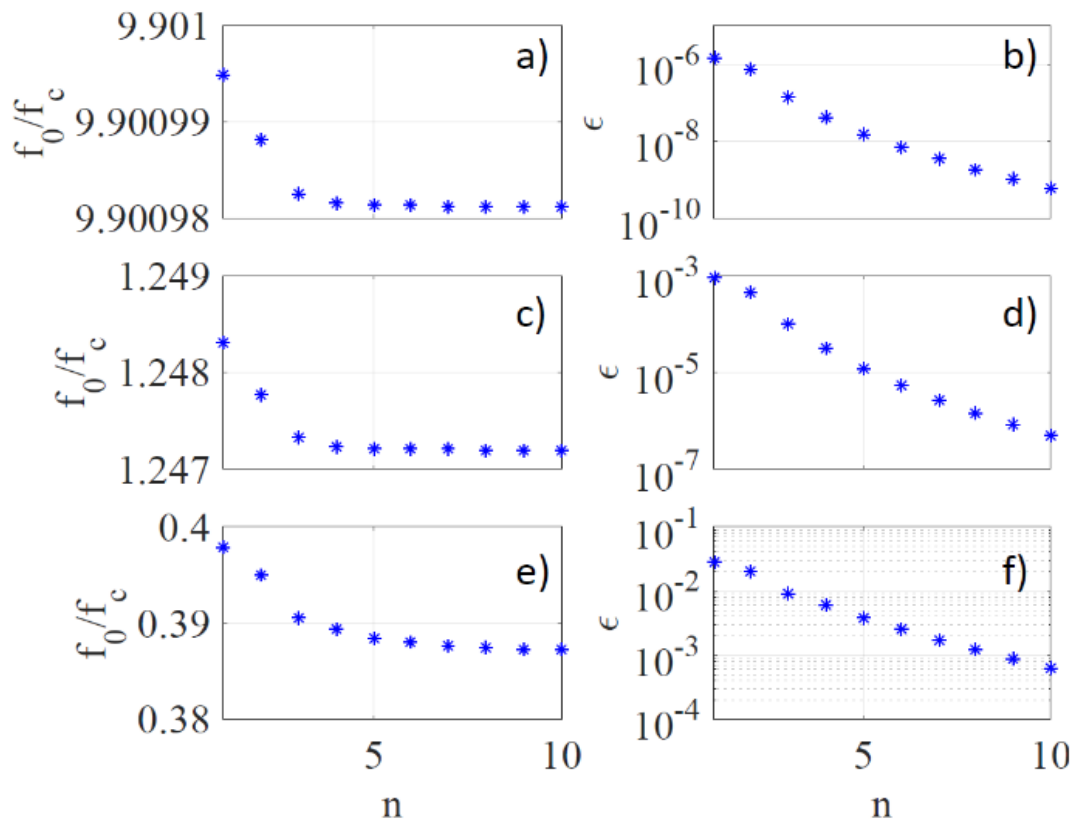

Figure 2: Left column plots: computation of $f_{0} / f_{c}$ as a function of $\mathrm{n}$; right-column semi-log plots: relative errors of $f_{0} / f_{c}$ for a) and b) $L / L_{c}=0.6$, c) and d) $L / L_{c}=2$, e) and f) $L / L_{c}=10$.

The nondimensional natural frequency $f_{0} / f_{c}$, where:

$f_{c}=\frac{1}{2 \pi} \sqrt{\frac{g}{L_{c}}}$

is related to the lowest eigenvalue of $A$ and is extracted from Eq. 10. It depends only upon the ratio $L / L_{c}$ and the size of the matrix $A$. To investigate the accuracy of numerical solution of Eq. 15, we compute $f_{0} / f_{c}$ for several $n$ values while $L / L_{c}$ is fixed. Figure 2 shows our results for $n=1$ to 10 for $L / L_{c}=0.6 ; 2$ and 10 . We have also calculated the relative error $\epsilon$ with respect to a reference value obtained by solving Eq.15 with $n=200$. We can see that, as expected, the error is low for small values of $L / L_{c}$ (see Figure 2a) and 2b)) because in this case, the plate weight can be neglected and the solution corresponds to Eq. 3. For larger $L / L_{c}$ values, a higher order Galerkin decomposition is needed to reach more accurate results. However, we observe that from a value as small as $n=6$, variations of $f_{0} / f_{\text {c }}$ are insignificant (Figure 2e)). Beyond this $n$ value, the relative error is lower than $0.3 \%$ as it can be seen in Figure $2 f$ ).

In order to have an idea of the critical value $n=n_{c}$ necessary to obtain a given relative error $\epsilon=$ $10^{-1}, 10^{-2}, 10^{-3}, 10^{-4}$ or $10^{-5}$ we plotted $n_{c}$ as a function of $L / L_{c}$ in Figure 3 . It can be observed that $n=1$ is sufficient to obtain a relative error equal to $10 \%$ for the whole explored length interval. An accuracy $\epsilon=1 \%$ is reached only if $n=2$ for $4.5 \leq L / L_{c} \leq 6$, and $n=5$ for $L / L_{c} \geq 16$. A higher accuracy requires $n_{c}>1$ as soon as $L / L_{c} \geq 0.5$. 


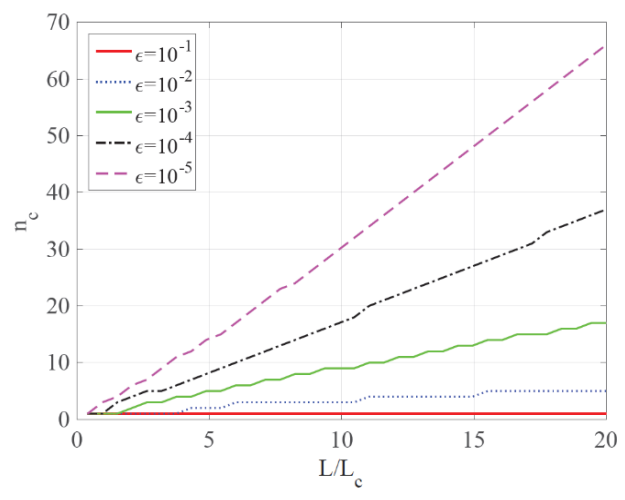

Figure 3: Evolution of the $n$ critical value $n_{c}$ required to reach a relative error equal to $\epsilon=10 \%$ (red straight line at $n_{c}=1$ ), $\epsilon=1 \%$ (blue dotted line), $\epsilon=0.1 \%$ (green plain line), $\epsilon=0.01 \%$ (black dashed dotted line) and $\epsilon=0.001 \%$ (magenta dashed line).

\section{EXPERIMENTS}

\subsection{Plate characteristics}

Experiments were performed with three different materials whose length was varied in order to access the power law of $f_{0}$ with respect to $L$. Plate 1 corresponds to a polyester plate while plates 2 and 3 were molded with silicon. Each plate flexural rigidity was determined by preliminary experiments which consist in measuring the cantilever plate's static deflection when it is submitted to its own weight. A photography of this method is shown in the inset of Figure 4 . In this case indeed, the free extreme of the plate is subjected to a transversal deflection $\delta$ equal to (Wilson and Lord Jr, 1973):

$\delta=\frac{m_{\ell} g L^{4}}{8 E I}$

In order to have a more accurate value, $\delta$ is measured for different free lengths $L$ values. The plot of $\delta$ vs. $L^{4}$ permits to perform a least-square linear fit whose coefficient is related to EI. Figure 4 shows the experimental points and the linear fits for the two silicon plates.

Table 1 shows the plate dimensions, their mass, the flexural rigidity, and the critical length calculated via Eq. 6.

\subsection{Methodology}

The experimental scheme is shown in Figure 5. The plate is clamped from its upper extreme while its bottom extreme is taken away from its equilibrium position and left free to oscillate. A motion sensor (Keyence LK-GD500) is connected to a computer and registers the free extreme position each 2 millisecond for plate 1 and each 2 ms for plates 2 and 3 . The spatial resolution is $0.3 \mathrm{~mm}$. The same procedure is repeated for different hanging lengths and for the three plates.

Figure 6a) shows the example of a registered signal. The Fourier spectrum calculated in Figure 6b) presents clearly a peak whose position provides the oscillation frequency $f_{0}$ for each plate length. The width at half-height, pointed out by a red line in Figure $6 \mathrm{~b}$ ), allows to estimate an errorbar for each frequency value.

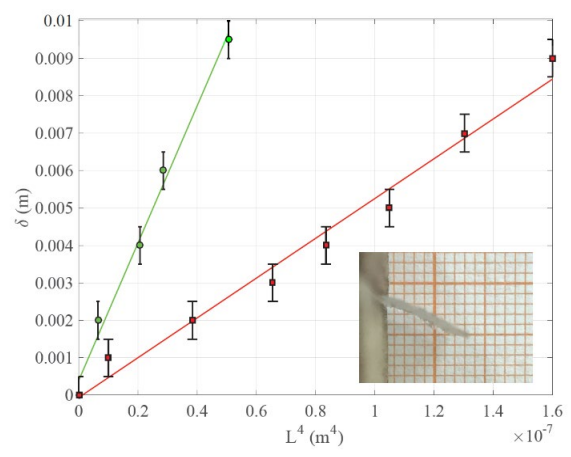

Figure 4: Experimental points of the static transversal deflection $\delta$ as a function of $L^{4}$ for $(\bullet)$ plate 2 , ( $(\mathbf{a})$ plate 3 . Lines show the linear fits from which flexural rigidity is extracted. Insert: photography of plate 2 deflected by its own weight. 
Table 1: Plate characteristics.

\begin{tabular}{cccc}
\hline & Plate 1 (polyester) & Plate 2 (thin silicon) & Plate 3 (thick silicon) \\
\hline$L_{\text {tot }}(\mathrm{cm})$ & 43.1 & 12.5 & 12.5 \\
$H(\mathrm{~cm})$ & 10.0 & 2.0 & 2.3 \\
$e(\mathrm{~cm})$ & 0.2 & 1.0 & 2.0 \\
$E I\left(\mathrm{~N} \cdot \mathrm{m}^{2}\right)$ & $2.1 \times 10^{-4}$ & $1.7 \times 10^{-7}$ & $1.3 \times 10^{-6}$ \\
$m_{\ell}(\mathrm{g} / \mathrm{m})$ & 26.1 & 24.8 & 57.0 \\
$L_{c}(\mathrm{~cm})$ & 9.0 & 0.87 & 1.4 \\
$f_{c}(\mathrm{~Hz})$ & 1.7 & 5.3 & 4.2 \\
\hline
\end{tabular}

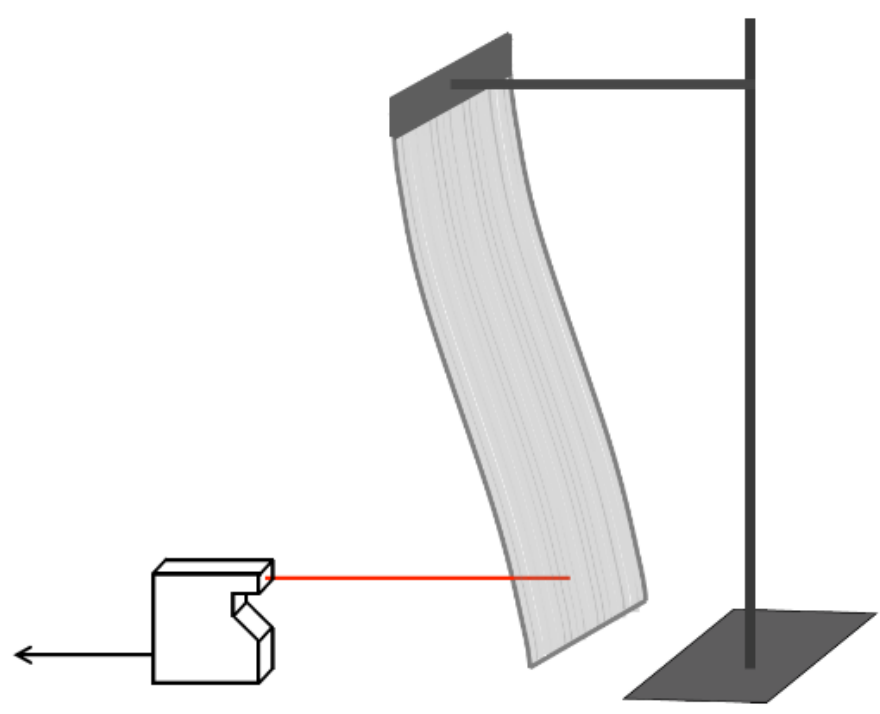

Figure 5: Experimental scheme used to extract the natural frequency of the flexible plate.
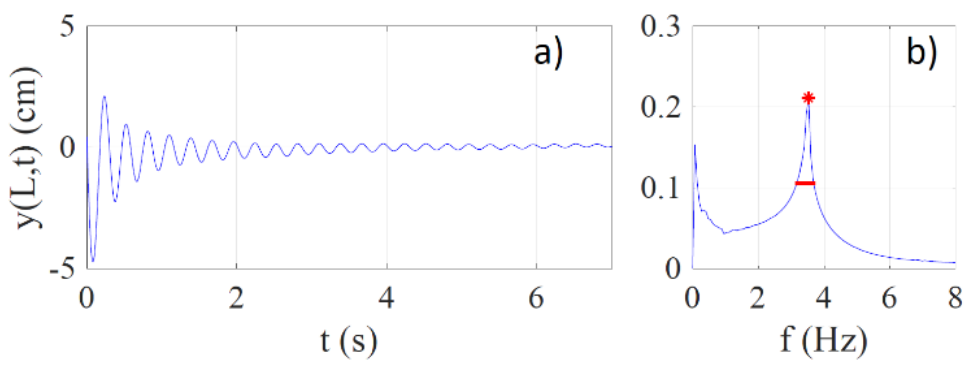

Figure 6: a) Oscillations of plate 1's free extreme for $L=13 \mathrm{~cm}$. b) Fourier spectrum. The red star points out the peak máximum while the red line at the peak mid-height permits to estimate the errorbar.

\section{RESULTS}

Raw frequency values are shown in the log-log plot of Figure 7 as a function of length for the three plates. The different lines represent the two limit cases: the three parallel colored, tilted dashed lines stand for the purely flexible theory (Eq. 3, $f_{0} \sim L^{-2}$ ) while the black dotted line points out the frequency evolution for a hanging chain (Eq. 4, $f_{0} \sim L^{-1 / 2}$ ). These straight lines intersect for $L=2.02 L_{c}$ and $f_{0}=0.70 f_{c}$. The experimental points of the more rigid plate (plate 3, blue triangles) are better described by Eq. 3 for the smallest lengths whereas they diverge from this law for longer plates. The most flexible plates (plates 2 and 3 , green circles and red squares) clearly behave as hanging chains for $L>L_{c}$. As expected, this limit case clearly does not depend upon the plate Young's modulus since the same dotted line represents a unique asymptotic behavior for all the plates. The intermediate experimental values $L \sim L_{c}$ are in agreement with the three theoretical plain colored lines. 


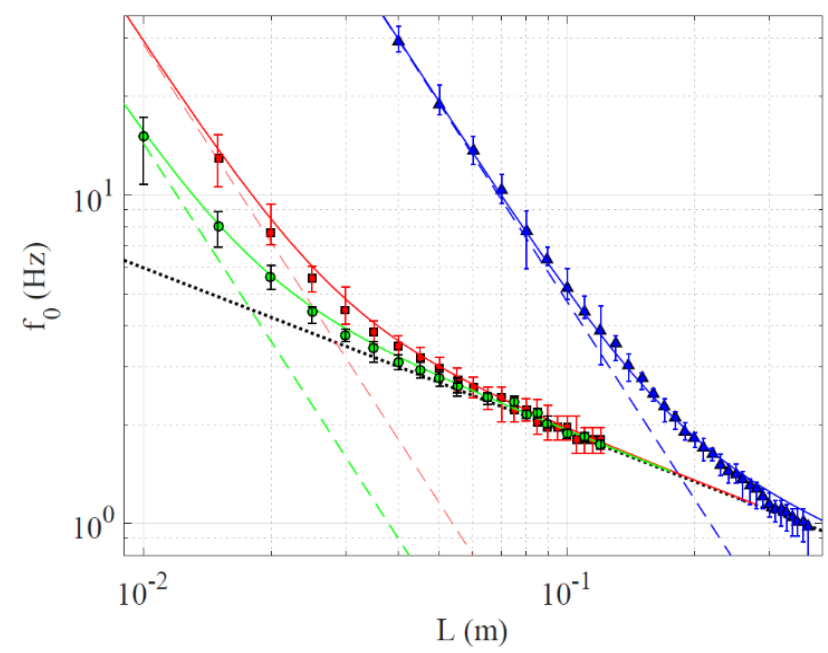

Figure 7: Experimental points for the three plates superimposed with the two limit cases and with the Galerkin expansion calculated for each plate. ( $\mathbf{\Delta})$ plate $1,(\bullet)$ plate 2, (a) plate 3. The three parallel colored dashed straight lines point out theoretical laws (Eq. 3) for each flexible plate, while the black dotted line denotes the hanging chain frequency (Eq. 4). The three curved colored lines stand for theory.

Finally, Figure 8 shows how all the experimental points can be superimposed by considering the critical length $L_{c}$ and critical frequency $f_{c}$ for each plate shown in Table 1. Errorbars were removed in order to get a clearer graphical visualization. The theoretical curve tends to the expected limit power laws $f_{0} \sim L^{-2}$ (dashed line) for $L \ll L_{c}$ and $f_{0} \sim$ $L^{-1 / 2}$ (dotted line) for $L \gg L_{c}$ whereas the intermediate values $L \sim L_{c}$ (thick gray curve) superimpose on the solution found by the Galerkin expansion.

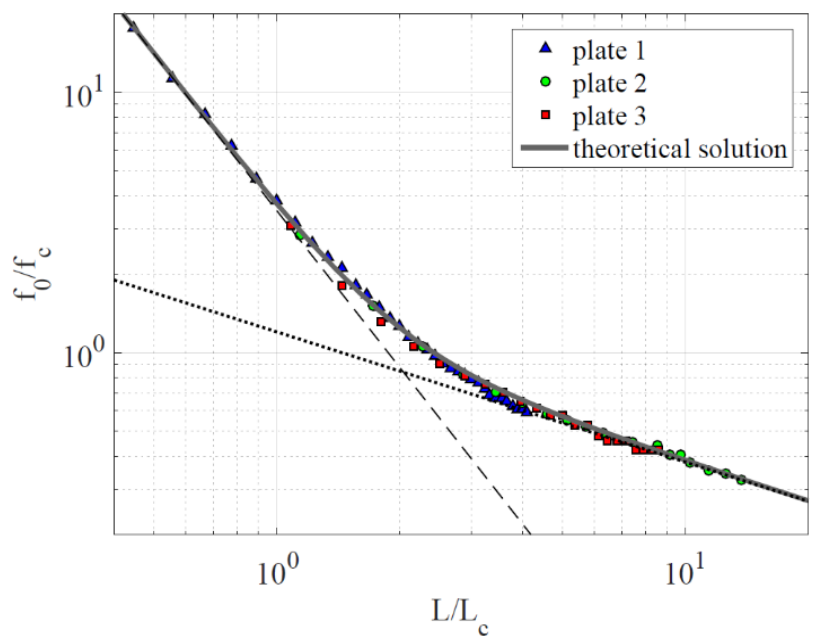

Figure 8: Experimental points for the three plates superimposed with theoretical curve where $L / L_{c}$ is in $x$ and $f_{0} / f_{c}$ in $y:(\boldsymbol{\Delta})$ plate $1,(\bullet)$ plate 2, (घ) plate 3. Dashed line: nondimensional version of the purely flexible plate (Eq. 3). Dotted line: nondimensional version of the hanging chain (Eq. 4). Plain thick gray line: Galerkin expansion.

\section{CONCLUSION}

This study is devoted to the modification of the flexible plate natural frequency when its weight cannot be neglected anymore. This occurs when the plate length $L$ reaches values greater than a critical length $L_{c}$. This latter quantity is expressed as a function of the ratio between the plate flexural rigidity and the weight per unit of length. In order to solve the complete differential motion equation, a Galerkin decomposition was performed using the spatial modes which develop for a purely flexible plate. We computed the number of modes necessary to get a fixed relative error: as expected, this number increases as $L$ is greater since in this case, the plate weight gets more and more important. Moreover, laboratory experiments were performed with different plates molded in three distinct materials and for 
various length values. The natural frequency $f_{0}$ is measured by recording the plate's free oscillations. The systematic exploration of a large length interval allows to visualize how the power law evolves when $L$ is varied. The data points tend to the two limit power laws $f_{0} \sim L^{-2}$ for $L / L_{c} \ll 1$ and $f_{0} \sim L^{-0.5}$ for $L / L_{c} \gg 1$ whereas the intermediate values $L \sim L_{c}$ are correctly fitted by our Galerkin expansion. All the experimental points superimpose on a same curve by considering a nondimensional parameter frequency and a nondimensional length $L / L_{c}$.

\section{ACKNOWLEDGMENTS}

J. R. I and A. C. acknowledge CONACyT and the Mexican Secretaría de la Educación Superior for supporting this project [grant number A1-S-55355].

Author's Contributions: Conceptualization, A Cros; Data curation, JG Rivas Iñiguez and A Cros ; Formal Analysis, ML Daza Torres, A Palafox González and A Cros; Funding acquisition, A Cros; Methodology, JG Rivas Iñiguez, ML Daza Torres, A Palafox González and A Cros; Writing - original draft, A Cros; Writing - review \& editing, ML Daza Torres, A Palafox González and A Cros.

Editor: Marcílio Alves

\section{References}

Cros, A., Arellano Castro, R.F. (2016). Experimental study on the resonance frequencies of a cantilevered plate in air flow. Journal of Sound and Vibration 363: 240-246.

De Langre, E., Doaré, O., Pellet, F. (2001). Force suiveuse critique sur une colonne pesante semi-infinie: modèle et expériences. Comptes Rendus de l'Académie des Sciences-Series IIB-Mechanics 329(3): 175-178.

Dungundji, J., Dowell, E., Perkins, B. (1963). Subsonic flutter of panels on continuous elastic foundations. AIAA Journal 1: 1146-1154.

Eloy, C., Souilliez, C., Schouveiler, L. (2007). Flutter of a rectangular plate. Journal of Fluids and Structures 23(6): 904-919.

Gregory, R., Païdoussis, M. P. (1966) Unstable oscillation of tubular cantilevers conveying fluid ii. experiments. Proceedings of the Royal Society of London. Series A. Mathematical and Physical Sciences 293(1435): 528-542.

Lamb, H. (1929). Higher Mechanics. Cambridge University Press.

Levinson, D.A. (1977). Natural frequencies of a hanging chain. American Journal of Physics 45(7): 680-681.

Newl, D.E. (2004). Pedestrian excitation of bridges. Proceedings of the Institution of Mechanical Engineers, Part C: Journal of Mechanical Engineering Science 218(5): 477-492.

Païdoussis, M.P., Des Trois Maisons, P.E. (1971). Free vibration of a heavy, damped, vertical cantilever. Journal of Applied Mechanics 38(2): 524-526.

Schäfer, B. (1985). Free vibrations of a gravity-loaded clamped-free beam. Ingenieur-archiv 55(1): 66-80.

Turvey, K. (1990). An undergraduate experiment on the vibration of a cantilever and its application to the determination of young's modulus. American Journal of Physics 58(5): 483-487.

Verbin, Y. (2014). Boundary conditions and modes of the vertically hanging chain. European Journal of Physics 36(1): 015005.

Wilson, F., Lord Jr, A. (1973). Young's modulus determination via simple, inexpensive static and dynamic measurements. American Journal of Physics 41(5): 653-656.

Xi, L.Y., Li, X.F., Tang, G.J. (2013) Free vibration of standing and hanging gravity-loaded rayleigh cantilevers. International Journal of Mechanical Sciences 66: 233-238.

Yokoyama, T. (1990). Vibrations of a hanging timoshenko beam under gravity. Journal of Sound and Vibration 141(2): 245258. 